\title{
АННОТАЦИЯ
}

Т Телью настоящей статьи является определение возможных подходов к разработке процесса «встроенной устойчивости», заключающейся в синхронизации стратегии организации с тенденциями устойчивого развития. Для этого на основе применяющегося на практике процесса «встроенной безопасности» (safety-by-design) предлагается процесс «встроенной устойчивости» (sustainability-by-design). В качестве ключевого звена процесса предлагается использовать систему индикаторов устойчивости, дополненную индикаторами риска и жизнестойкости. Для усиления адаптивного потенциала компаний в области устойчивого развития процесс формирования «встроенной устойчивости» дополнен включением демократических процедур путем структурированного участия заинтересованных сторон. В результате предлагаемый процесс сочетает жесткую структуру ценностей и целей с «мягкой настройкой» в форме демократических процедур. Это позволит компаниям не только снизить неопределенности, присущие проблеме запаздывающего регулирования, но и сформировать среду для разработки наиболее эффективных альтернатив развития компаний в области устойчивости.

\section{КАЮЧЕВЫЕ CАOBA:}

устойчивое развитие, управление риском, жизнестойкость.

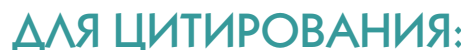

Саченко Л.А. (2020). Формирование «встроенной устойчивости» организаций с использованием расширенной системы индикаторов риска при принятии стратегических решений // Стратегические решения и риск-менеджмент. Т. 11 . № 4. С. $402-411$. DOI: $10.17747 / 2618-947 X-2020-4-402-411$. 


\title{
Implementing \\ "sustainability-by-design" \\ approach in organizations by using an extended risk indicators system for making strategic decisions
}

L.A. Sachenko ${ }^{1}$

1 "Risk-profile" LLC

\begin{abstract}
The purpose of this article is to identify possible approaches to the development of the "sustainability-by-design" process, which

1 consists in synchronizing the organization's strategy with the trends of sustainable development. For this purpose, a process of "sustainability-by-design" is proposed on the basis of the "safety-by-design" process applied in practice. As a key link in the process, it is proposed to use a system of sustainability indicators, supplemented by indicators of risk and resilience. To strengthen the adaptive capacity of companies in the field of sustainable development, the process of building "sustainability-by-design" is complemented by the inclusion of democratic procedures through structured stakeholder participation. As a result, the proposed process combines a rigid structure of values and goals with a "soft setup" in the form of democratic procedures. This will allow companies not only to reduce the uncertainties inherent to the pacing problem, but also to create an environment for the promotion of the most effective alternatives to the development of companies in the field of sustainability.
\end{abstract}

\section{KEYWORDS:}

sustainable development, risk management, resilience.

\section{FOR CITATION:}

Sachenko L.A. (2020). Implementing "sustainability-by-design" approach in organizations by using an extended risk indicators system for making strategic decisions. Strategic Decisions and Risk Management, 11(4), 402-411. DOI: 10.17747/2618-947X-2020-4-402-411. 


\section{BBEAЕHИЕ}

Влияние тенденций устойчивого развития на экономическую деятельность становится все значительнее. В то же время существует высокая неопределенность как состава возможных требований в будущем, так и соответствующих им последствий. В таких условиях процесс стратегического планирования относительно устойчивости напоминает стрельбу по движущейся мишени, а опора на текущие требования к отчетности по устойчивому развитию может привести к принятию ошибочных и даже фатальных стратегических решений. Как бизнесу обеспечить соответствие меняющимся требованиям и сформировать четкую позицию в отношении устойчивого развития?

Для решения этой задачи в статье анализируются возможные подходы и предлагаются решения по адаптации стратегии организаций к тенденциям устойчивого развития.

Вначале проведен анализ текущей ситуации по требованиям и тенденциям в области устойчивого развития. Проиллюстрировано наличие проблемы запаздывающего регулирования [Downes, 2009; Marchant et al., 2011], которая проявляется в периоды быстрого технологического развития, а также показаны признаки смены модели ведения бизнеса. Далее в разделе рассмотрены подходы к решению проблемы запаздывающего регулирования в областях деятельности, связанных с безопасностью [Yatsalo et al., 2005; Strategic environmental assessment.., 2018; Trump et al., 2020]. Показано, что процесс формирования «встроенной безопасности» может быть взят за основу при формировании «встроенной устойчивости» при условии устранения выявленных ограничений. В четвертом разделе выполнен обзор имеющихся индикаторов в области устойчивости [Hardi, Zdan, 1997; Wu, $\mathrm{Wu}, 2012]$, а также обоснована целесообразность дополнения системы индикаторов устойчивости индикаторами риска и жизнестойкости [Sikula et al., 2015; Faber, 2018; GillespieMarthaler et al., 2019]. Пятый раздел посвящен модификации процесса формирования «встроенной безопасности» для целей устойчивости. В качестве ключевого компонента процесса предлагается использовать расширенную систему индикаторов устойчивости, риска и жизнестойкости. Кроме того, для восполнения пробелов, присущих технократической системе индикаторов [Reid, Rout, 2020], в процесс включены демократические процедуры. Также предложен вариант использования расширенной системы индикаторов одновременно в двух направлениях: как для исключения неэффективных альтернатив, так и для формирования альтернатив в области устойчивости.

\section{2. РИСКИ АИЯ БИЗНЕСА, СВЯЗАННЫЕ С ИЗМЕНЕНИЕМ MOАЕАИ УСТОЙЧИВОСТИ}

Проблемы устойчивого развития в последние годы занимают все большее место в деятельности компаний. Установка на рост экономики привела к ряду серьезных проблем, требующих достаточно оперативного реагирования всего мирового сообщества. В частности, остро проявляются экологические проблемы, изменение климата, социальные проблемы. Международное сообщество отреагировало принятием в 2015 году нескольких базовых документов: Парижского соглашения в рамках Рамочной конвенции ООН об изменении климата ${ }^{1}$, Сендайской рамочной программы по снижению риска бедствий на 2015-2030 годы ${ }^{2}$, программы ООН по целям устойчивого развития (ЦУР) - от преодоления нищеты и ликвидации голода до обеспечения гендерного равенства и принятия срочных мер по борьбе с изменением климата.

Бизнес, являясь важнейшей частью мировой жизнедеятельности, также активно вовлекается в процессы по обеспечению устойчивости. На сегодняшний момент эта деятельность, казалось бы, прямо не регламентирована и не координируется. Для большинства компаний устойчивое развитие означает ряд проектов, направленных на комплаенс и репутационные цели. Важным направлением этой деятельности является подготовка соответствующей отчетности и получение рейтингов. Таким образом, имеется достаточно высокая степень свободы как относительно самой деятельности по устойчивому развитию, так и по подготовке отчетности. Например, наиболее часто применяемое на практике Руководство по отчетности в области устойчивого развития организации Global Reporting Initiative (GRI G4) ${ }^{3}$ не содержит четко установленных требований к составу раскрываемой информации, имеется лишь базовый перечень рекомендуемых аспектов и показателей для их раскрытия. Методика же оценки существенности тем, определенных для компании с целью внесения в отчет, по сути, произвольна и позволяет показать только наиболее выигрышные направления деятельности.

На усмотрение компаний также оставляется вопрос полноты раскрытия всех этапов жизненного цикла производства продукции или услуг. Это приводит к тому, что оценки по жизненному циклу достаточно слабо представлены в большинстве отчетов [Stewart et al., 2018]. Разный уровень раскрытия информации наблюдается в отчетах и по цепочке поставок: от краткого описания закупок и основных поставщиков до достаточно полного представления внедрения практик устойчивого развития по цепочке поставок.

Интерес к проблематике устойчивого развития в деловом мире растет как с точки зрения финансовых институтов и инвесторов, так и со стороны деловых партнеров. Для более системного представления данных об уровне устойчивости и перспективности компаний широко используются ESG-рейтинги (E - environmental, S - social и G - governance - экологический, социальный и управленческий соответственно). При расчете рейтинга применяются определенным образом взвешенные метрики, которые основаны на принципах сопоставимости, воздействия, доступности данных и отраслевой значимости для каждой отраслевой группы. Предполагается, что ESG-рейтинги представляют собой более объективный источник информации, чем отчеты компаний по устойчивому развитию. Действительно, расчеты рейтингов выполняются по идентичному алгоритму для всех компаний и могут

\footnotetext{
${ }^{1}$ Парижское соглашение РКИК ООН. 12 декабря 2015 года.

${ }^{2}$ Сендайская рамочная программа по снижению риска бедствий на 2015-2030 годы. UNISDR, 2015.

${ }^{3}$ URL: https://www.globalreporting.org/. 
служить основой для сопоставительных оценок. Однако существует большая зависимость результата как от методики оценки, так и от наличия данных по конкретному региону. Среди российских ESG-рейтингов можно упомянуть RAEXEurope $^{4}$, а также индексы РСПП по устойчивому развитию 5 .

В последнее время растет интерес инвесторов к импакт-инвестированию. В целом оно созвучно устойчивому инвестированию, однако в большей степени направлено на выявление возможных компаний-лидеров по решению глобальных проблем.

Именно импакт-инвестирование ближе к сути происходящей смены модели ведения бизнеса. Устойчивость в глобальном смысле перестает быть объектом комплаенса и источником косвенных выгод. Теперь устойчивость становится прямым источником роста прибыли и укрепления конкурентных позиций. При этом требования к устойчивости перестают быть внешними по отношению к компаниям, а становятся органичной и неотъемлемой внутренней потребностью бизнеса. И дело не только в количественных изменениях: объем рынка только климатических инвестиций, по некоторым оценкам ${ }^{6}$, достигнет к 2030 году 23 трлн долл.

Совершенно очевидно, что происходящая трансформация всего мирового уклада, хотя и имеет вполне определенные цели и предполагаемые пути их достижения, связана с аномально высокой неопределенностью и динамикой. Стратегии, основанные на экстраполяции и анализе предыдущего опыта, становятся резко недостаточными. Компаниям нужны средства адаптации к непредвиденным изменениям для сохранения своей стратегической устойчивости.

\section{3. «ВСТРОЕННАЯ БЕЗОПАСНОСТЬ» КАК ОСНОВА ФОРМИРОВАНИЯ «ВСТРОЕННОЙ УСТОЙЧИВОСТИ»}

Прежде чем перейти к описанию основных подходов к формированию «встроенной устойчивости», уточним терминологию. Определение «встроенной устойчивости» компаний, используемое в контексте настоящей статьи, не тождественно понятию стратегической устойчивости [Самосудов, 2006; Григорьева, 2013], в общем случае понимаемой как «взаимодействие компонентов организации, которое позволяет обеспечить положительную динамику показателей деятельности для повышения эффективности функционирования организации в длительный период» [Кузнецова, 2020]. «Встроенная устойчивость» для целей настоящей статьи это свойство, приобретаемое компаниями в результате процесса адаптивной синхронизации с основными мировыми тенденциями, связанными с устойчивым развитием.

Переход на новую модель принятия стратегических решений в свете изменений в области устойчивого развития необходим в силу обострения так называемой проблемы запаздывания (pacing problem) [Downes, 2009; Marchant et al., 2011]. Суть проблемы запаздывания заключается в том, что темп технологического развития в последние годы значи-
Рис. 1. Проблема запаздывающего регулирования

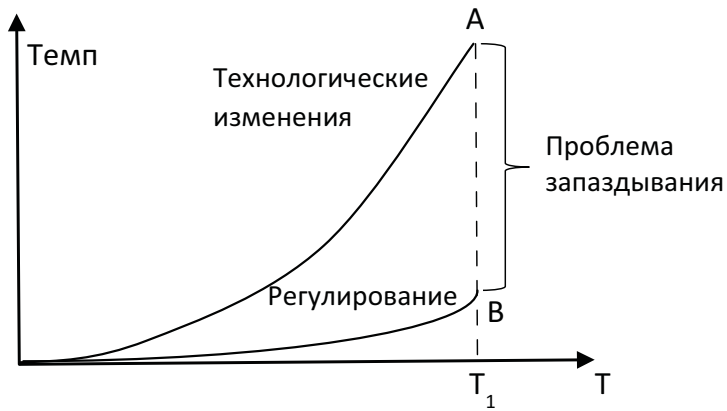

Источник: [Marchant, 2011].

тельно выше, чем способность регулирующих органов обеспечивать адекватные рамки для происходящих изменений. По этой причине для компаний складывается ситуация кажущейся свободы и несистемности в области регулирования устойчивого развития, которая описана в предыдущем разделе. Если обратиться к графической интерпретации, то сегодняшняя ситуация выглядит как проблема запаздывающего регулирования (рис. 1).

При этом компания, находящаяся в момент времени $\mathrm{T}_{1}$ в точке А, при принятии стратегических решений опирается на регулирующие документы в объеме В. Этот регуляторный зазор АВ может создать иллюзию относительной свободы и привести к принятию неэффективных решений.

В целях снижения степени неопределенности некоторыми зарубежными регулирующими органами ${ }^{7}$ в области нанотехнологий рекомендовано использование метода «встроенной безопасности» (safety-by-design) при рассмотрении стратегических альтернатив. Авторы работы [Trump et al., 2020] предлагают воспользоваться методом мультикритериального анализа принятия решений (MCDA), при этом в качестве дополнительной опоры авторы предлагают использовать материалы по оценке рисков, накопленные на текущий момент времени (рис. 2), снижение неопределенности при принятии решения с запаздывающим регулированием. Это позволяет существенно снизить неопределенность до отрезка АС и быстрее двигаться в направлении адекватного регулирования.

Рис. 2. Снижение неопределенности при принятии решения с запаздывающим регулированием

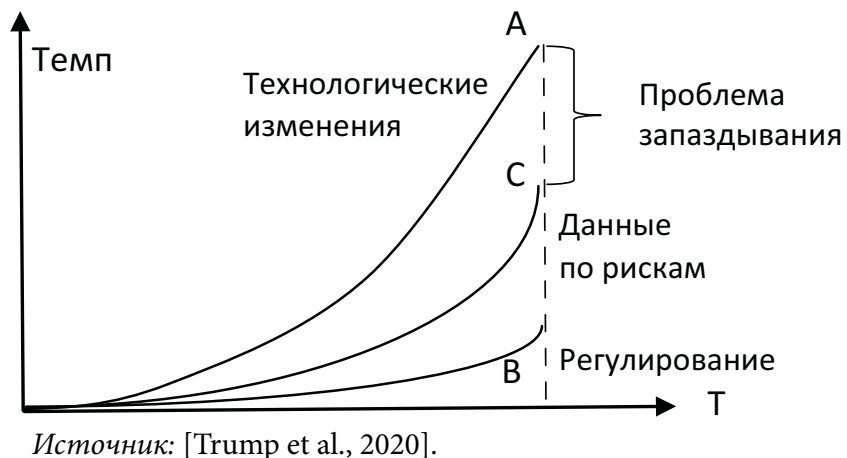

Источник: [Trump et al., 2020].

${ }^{4}$ URL: https://raexpert.eu/esg_corporate_ranking/.
${ }^{5}$ URL: https://media.rspp.ru/document/1/8/b/8bc5ac4b5914eddd2eb4f34ee695d550.pdf

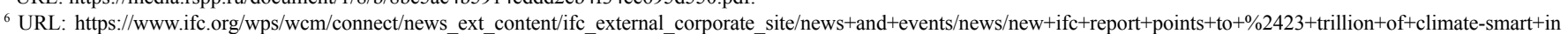
vestment + opportunities + in + emerging + markets + by +2030 .

${ }^{7}$ Например, National Institute for Occupational Safety and Health (NIOSH), а также European Union's Horizon 2020 Programme (цит. по [Trump et al., 2020]). 
Рис. 3. Схема процесса «встроенной безопасности»

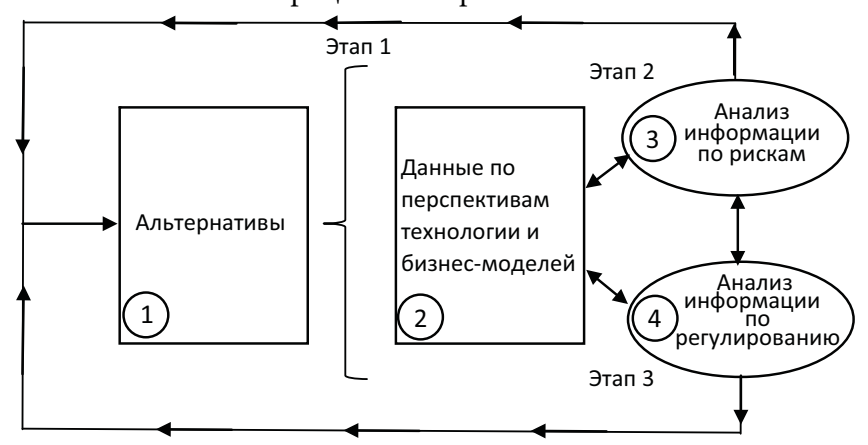

Источник: [Trump et al., 2020].

В то же время компаниям выгодно иметь опору на те же самые данные по оценке рисков. При этом, помимо более точного прогноза регуляторных требований, они будут иметь выигрыш во времени, а также в информации для принятия стратегических решений.

Первый этап процесса «встроенной безопасности», представленного на рис. 3, предполагает анализ альтернатив развития технологии, бизнес-модели, заинтересованных сторон (стейкхолдеров) и их интересов, а также возможные альтернативы в области устойчивого развития. Второй этап заключается в проведении сравнительной оценки наиболее предпочтительных альтернатив, выявленных на первом этапе, с текущим срезом информации по рискам. На третьем этапе рассматриваются приоритетные альтернативы сквозь призму текущего регулирования. Анализ на этом этапе должен предполагать выбор тех альтернатив, реализация которых связана с наибольшими выгодами, наименьшими рисками и наименьшими затратами на комплаенс.

Сама представленная схема не является единственно правильной, а может видоизменяться в зависимости от контекста задачи. Например, в задачах управления экологическими рисками [Yatsalo et al., 2005] возможные подходы к анализу альтернатив представлены на базе методологии DECERNS (Decision Evaluation in Complex Risk Network Systems). Один из примеров наиболее взвешенного комплексного подхода как к организации самого процесса, так и к выбору основных методов и критериев изложен в рекомендациях МАГАТЭ «Стратегическая экологическая оценка ядерных энергетических программ: руководящие принципы», где подробно рассматриваются цели, обязательные компоненты процесса, а также спектр возможных методов и критериев анализа, их достоинств и недостатков [Strategic environmental assessment.., 2018]. Такой серьезный подход абсолютно оправдан для капиталоемких объектов атомной энергетики.

Для тех организаций, которые основной упор в своей стратегии делают не на качество планирования, а на высокую адаптивность, больше подойдет модель оценки жизнестойкости MIRA, разработанная для военных установок [Sikula et al., 2015]. Для перехода к адаптивной модели от командно-контрольной разработана методология SPARK [Dos Santos, Partidário, 2011], расшифровывающаяся как методология стратегического планирования для поддержки жизнестойкости.
Наряду с выводом об очевидной пользе от использования процессов типа «встроенной безопасности» для решения проблемы запаздывания необходимо выделить два ключевых момента, требующих доработки для применения в целях «встроенной устойчивости»:

- во-первых, прямая замена индикаторов риска на индикаторы устойчивости в блоке 3 на рис. 3 , представленные прогнозом регулирования в области устойчивости, явно недостаточна ввиду высокой степени неопределенности тенденций устойчивого развития;

- во-вторых, процесс «встроенной безопасности» ставит своей целью только отсев опасных альтернатив. Таким образом, прямой перенос процесса в область устойчивости решит только проблему запаздывающего регулирования. При этом влияние тенденций устойчивости на компанию будет учтено, а влияние самой компании на внешнюю среду - нет. Тем самым исключается возможность планирования проактивных действий компании в области устойчивости.

Даже модель МАГАТЭ, предполагающая использование положительных моментов технологии, в кратком описании описывает свою основную функцию как роль «критичного друга» [Strategic environmental assessment.., 2018].

Исходя из анализа достоинств и недостатков методов «встроенной безопасности», можно сделать вывод о том, что они могут быть взяты за основу для применения в задачах стратегического планирования, касающихся устойчивого развития. Однако для этого недостаточно механически заменить «риски» на «устойчивость» - необходимо устранить выявленные ограничения, что и будет предметом следующих разделов.

\section{4. ПОАХОАЫ К РАЗРАБОТКЕ И ПРИМЕНЕНИЮ РАСШИРЕННОЙ СИСТЕМЫ ИНАИКАТОРОВ УСТОЙЧИВОГО РАЗВИТИЯ}

Ключевым элементом процесса принятия решений является блок 3 (рис. 3), связанный с анализом информации по устойчивости. Если целевое назначение процесса не просто комплаенс, а разработка стратегии компании, то предлагается представить этот блок не одним, а тремя совместными элементами:

- анализ информации по устойчивости (sustainability);

- анализ рисков;

- анализ жизнестойкости (resilience).

Логика такого сочетания объясняется тем, что для планирования адаптивной системы недостаточно только информации по перспективам в области устойчивости, где неопределенность и динамика изменений очень высоки. Для повышения надежности решений перспективно-аналитический блок по устойчивости должен быть дополнен блоком анализа рисков. Но в силу того, что классический риск-менеджмент достаточно эффективен лишь при незначительных отклонениях факторов риска от наблюдаемых ранее значений, необходим ввод компонента жизнестойкости, отвечаю- 
щего за анализ готовности адекватно реагировать на маловероятные и подчас непредсказуемые события.

В таком сочетании эти три области знаний: риск-менеджмент, устойчивость и жизнестойкость - в научной литературе стали встречаться сравнительно недавно. Тем не менее исследование [Nielsen, Faber, 2019] отмечает значительный рост числа публикаций и интереса и к каждой из этих тем, и к их совместному применению.

Подтверждение этому можно найти в самом определении устойчивости, как оно приведено в [Wu, Wu, 2012]: «Устойчивость отражает нашу способность поддерживать взаимосвязанную систему человек - природа в желательном состоянии в течение нескольких поколений в присутствии антропогенных и экологических возмущений и неопределенностей». То есть устойчивость поддерживается за счет двух основных составляющих - направленного движения к устойчивости и готовности сохранять ее в период шоков и кризисов. Именно поэтому для достижения устойчивости необходим риск-менеджмент, обладающий прекрасными возможностями для содействия достижению поставленных целей. Однако взаимосвязь между антропогенной и природной системами настолько сложна и непредсказуема, что обычная предиктивная аналитика риск-менеджмента не сможет отразить всех возможных вариантов развития событий. Поэтому в неменьшей степени для достижения устойчивости необходима жизнестойкость, которая занимается подготовкой к реагированию на непредвиденные события.

Методы традиционного риск-менеджмента все чаще оказываются не способны обеспечить компаниям адекватную реакцию на неблагоприятные события [Саченко, 2020]. Для восполнения этого пробела все приведенные выше концепции устойчивого развития должны быть дополнены анализом жизнестойкости. В научной литературе и практической деятельности актуальность этого направления за последнее время существенно возросла, и все чаще жизнестойкость рассматривается именно как комплементарная по отношению к управлению риском деятельность [Linkov, Trump, 2019; Sachenko, 2020]. Как и в других динамично развивающихся областях исследований, существуют различные подходы к пониманию и оценке жизнестойкости. Определение жизнестойкости, обобщающее все эти подходы, используется в Стратегии ООН по снижению риска стихийных бедствий ${ }^{8}$ «Способность системы, сообщества или общества, подвергающегося опасным воздействиям, к сопротивлению, поглощению, адаптации и восстановлению после них, своевременному и эффективному устранению последствий той или иной опасности, в том числе через сохранение и восстановление его сущностных свойств, основных структур и функций». То есть жизнестойкость обеспечивает второй компонент устойчивости - управление организацией под воздействием негативных эффектов, вызванных природными и антропогенными факторами.

Для практического применения описанного подхода по совместному анализу устойчивости, рисков и жизнестойкости необходима разработка единой системы индикаторов, объединяющих все три раздела такого анализа в свете целей и характера деятельности конкретной компании. К сожалению, универсального набора индикаторов для такой сложной задачи не существует. Поэтому требуется адаптация имеющихся методических предложений для конкретных задач.

Разработке индикаторов риска, устойчивости и жизнестойкости в последнее время посвящена масса исследований. Опуская хорошо известную практикующим специалистам тему индикаторов риска, приведем краткий обзор по индикаторам устойчивости, жизнестойкости отдельно и в перекрестных вариантах.

Индикаторы устойчивости предоставляют информацию о состоянии, динамике и основных движущих силах системы «организация - внешняя среда». Среди наиболее часто рекомендуемых для разработки критериев устойчивости являются так называемые принципы Белладжио, предложенные группой специалистов по устойчивости на конференции в итальянском Белладжио [Hardi, Zdan, 1997]. Они затрагивают весь процесс разработки и применения индикаторов устойчивости - от постановки целей и определения охвата задачи до оценки и улучшений. Конкретные примеры применения индикаторов устойчивости описаны для различных отраслей, в частности сталелитейной [Arena, Azzone, 2010], энергетической [Afgan et al., 2000], сельскохозяйственной [Mohamed et al., 2014]. Однако, как подчеркивается в [Wu, $\mathrm{Wu}, 2012]$, одном из наиболее подробных обзоров по индикаторам устойчивости, в зависимости от выбора системы индикаторов, их достаточности или избыточности, от задания граничных условий можно прийти к совершенно различным выводам относительно устойчивого развития. Главным фактором успеха при разработке адекватной системы индикаторов устойчивости будут наиболее полный учет принципов устойчивого развития во всей их сложности и понимание рамок, соответствующих этим критериям, выраженные в максимально емком виде.

Индикаторам жизнестойкости компаний посвящено значительное количество исследований, в частности [Bruneau et al., 2003; Lengnick-Hall et al., 2011; Rose, Krausmann, 2013]. Авторы работы [Linkov et al., 2019] выделяют четыре исчерпывающих компонента любой сложной системы: физический, информационный, когнитивный, социальный. В результате их объединения с процессом жизнестойкости авторами получена так называемая матрица жизнестойкости, которая учитывает качество каждого из выделенных жизненно важных компонентов системы на основных этапах обеспечения жизнестойкости: подготовки, поглощения шока, восстановления и адаптации. В работе [Ganin et al., 2016] представлен подход количественной оценки жизнестойкости системы на основе понятия критической функциональности, которая представляет собой минимально необходимый набор функций в период кризиса. Одна из наиболее методически завершенных работ по внедрению системы ранних предвестников, позволяющих предупредить серьезные инциденты и повысить организационную жизнестойкость, описывает метод REWI (Resilience based early warning indicators) [Qien et al., 2012). Он основан на оценке восьми составляющих жизнестойкости: понимании риска, предвидении, внимании, реагировании, робастности, обеспеченности ресурсами / достаточности быстроты реагирования, поддержки решений и дублирования (резервирования).

\footnotetext{
${ }^{8}$ Terminology on disaster risk reduction. United Nations international strategy for disaster risk reduction (2009). Geneva: United Nations.
} 
Помимо обособленных систем индикаторов по отдельным направлениям в последнее время все больше работ направлены на получение совместных оценок по рискам и жизнестойкости либо по жизнестойкости и устойчивости. Так, в работе [Sikula et al., 2015] предложена совместная система индикаторов риска и жизнестойкости MIRA (Military installation resilience assessment model), которая фокусируется на анализе взаимосвязей и функциональности и целостной оценки социотехнических систем и предназначена для повышения качества реагирования и адаптации на неблагоприятные события. Как пишут авторы, система не может быть все время устойчива ко всему, поэтому ключевым элементом анализа является определение критической функциональности, которую необходимо поддерживать. В работе [Gillespie-Marthaler, 2019] представлен набор индикаторов и метрик, относящихся к сравнительно новой концепции устойчивой жизнестойкости (sustainable resilience). Концепция направлена на повышение жизнестойкости системы, исходя из временно́го анализа уязвимостей и запаса прочности ввиду возможных угроз. Предполагается, что устойчивая жизнестойкость приобретается за счет способности системы претерпевать необходимые изменения. Как и в предыдущем примере [Sikula et al., 2015], базовым приоритетом в системе индикаторов является выживание системы, затем следуют индикаторы благосостояния, и на последнем уровне по значимости находятся индикаторы готовности к реагированию на инциденты. М. Фабер [Faber, 2018] приводит решение, балансирующее для социотехнической системы устойчивость, риски и жизнестойкость. Он пишет о том, что до недавнего времени устойчивость и жизнестойкость являлись разными областями знаний, однако, по его мнению, наступил момент, когда эти области объединены едиными источниками риска: провалы в природной системе автоматически ведут к провалам в социоэкономических системах, и наоборот.

Таким образом, на сегодняшний момент существует явно выраженная направленность на совмещение индикаторов устойчивости, рисков и жизнестойкости, которые представлены рядом методических разработок. Такое совмещение может способствовать принятию наиболее информированных и, следовательно, наиболее эффективных решений по развитию компаний в направлении устойчивости.

\section{5. ПРОЦЕСС ФОРМИРОВАНИЯ}

\section{«ВСТРОЕННОЙ УСТОЙЧИВОСТИ» КОМПАНИЙ}

Основной задачей при формировании «встроенной безопасности» является блокировка опасных путей развития технологий или систем. Для такой постановки задачи схема процесса, представленная на рис. 3, является одним из наиболее подходящих вариантов решения.

Однако при планировании стратегических решений относительно устойчивого развития постановка задачи существенно меняется. На настоящий момент среда ведения бизнеса претерпевает динамичные и недостаточно предсказуемые изменения. Прежде всего это связано с серьезной трансформацией ключевых показателей эффективности для компаний. Очевидно, что целевые показатели компаний не
Рис. 4. Схема процесса формирования «встроенной устойчивости» компании

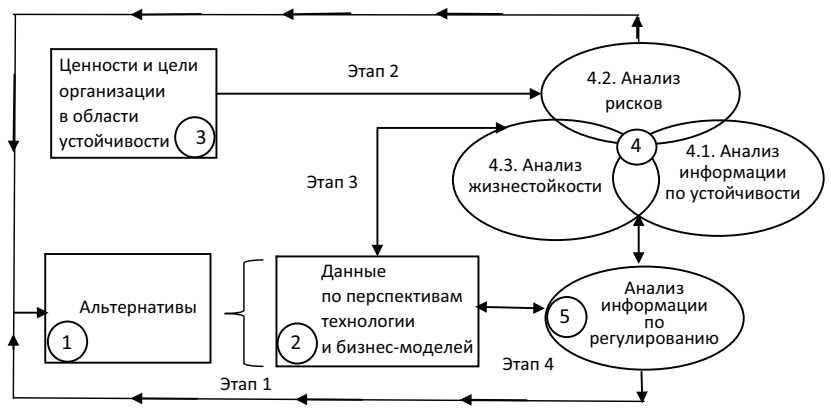

соответствуют общественному запросу, поэтому в ближайшее время неизбежно произойдет переход от чисто экономических показателей к некой комбинации экономической эффективности и устойчивости. Как конкретно сбалансируются в итоге эти цели деятельности для компаний, пока не ясно.

В таких условиях для формирования «встроенной устойчивости» недостаточно заблокировать не соответствующие критериям варианты, необходимо сформировать альтернативы, мягко «настраивающие» бизнес на волну устойчивого развития. Поэтому в плане созидания ограничительная функция рассмотренного ранее процесса явно недостаточна. Восполнить этот недостаток и сформировать инкубатор идей для устойчивого развития организации возможно, несколько модифицировав схему процесса, представленную на рис. 3, в схему, представленную на рис. $4,-$ схему процесса формирования «встроенной устойчивости» компании.

В данной схеме блок индикаторов представлен уже тремя совместными разделами (4.1-4.3) по устойчивости, рискам и жизнестойкости. Однако решением проблемы запаздывающего регулирования выгоды от применения единой системы индикаторов по рискам, устойчивости и жизнестойкости не исчерпываются. Не менее важной, а возможно, и более важной функцией данного блока знаний является возможность его использования для генерации альтернатив для компании, касающихся устойчивого развития (этап 2 на рис. 4). Дело в том, что в последнее время практика применения систем индикаторов подвергается серьезной критике. Так, Дж. Рейд и M. Рут [Reid, Rout, 2020] приводят недостатки так называемого технократического подхода к разработке индикаторов устойчивости, основанного на переносе методов измерения технических систем на сложные социально-экономические системы. При этом переносе и впоследствии в процессе агрегирования индикаторов теряются существенные свойства и взаимосвязи, заложенные в таких системах, что отклоняет компанию от принятия эффективных решений. Кроме того, технократические оценки тяготеют к количественному выражению, что также не всегда соответствует сущностным свойствам социальных и природных систем. Для исправления этого недостатка авторы предлагают альтернативный подход к разработке системы индикаторов, основанный на широком участии заинтересованных сторон при обеспечении радикальной прозрачности. Ключевыми компонентами такой прозрачности выделяют ценностно-ориентированную прозрачность, информационную прозрачность при разработ- 
ке индикаторов, информационную прозрачность по рискам и устойчивости, прозрачность по целям в области устойчивого развития. При всей сложности и несовершенстве демократической процедуры с участием многих заинтересованных сторон такой подход позволит восполнить пробелы, которые могут быть допущены группой экспертов при разработке технократической системы индикаторов.

Помимо описанного в [Reid, Rout, 2020] назначения, а именно «мягкой настройки» системы индикаторов, демократическая процедура представляет еще одну возможность для компании по развитию в области устойчивости. Очевидно, что демократический подход может быть использован не только для коррекции системы индикаторов риска, но и для разработки самих альтернатив (этап 2 на рис. 4), тем самым обогащая возможности компании различными вариантами развития, выходящими за ограниченные представления узкой группы лиц.

Например, компания, четко обозначающая свои ценности и цели в области устойчивого развития, может стать привлекательной для наиболее ответственных в этом отношении сотрудников. При правильном понимании целей и специфики деятельности компании креативные сотрудники могут оказаться прекрасным источником альтернатив, ведущих к конкурентному преимуществу. Таким образом, дополнив использование расширенной системы индикаторов (этап 3 на рис. 4) четкой постановкой целей и организацией участия заинтересованных сторон (этап 2 на рис. 4), компания может не только усовершенствовать саму жесткую систему индикаторов, но и сформировать «питательную среду» для формирования альтернатив. Это позволит максимально эффективно организовать процесс адаптивной синхронизации с процессами устойчивого развития, обеспечивая компании «встроенную устойчивость».

\section{6. ЗАКАЮЧЕНИЕ}

Предложенная в статье схема процесса формирования «встроенной устойчивости» стратегии компании к процессам, связанным с устойчивым развитием, основана на анализе и нахождении баланса между двумя разнонаправленными эффектами:

- влияние процессов, касающихся устойчивого развития, на организацию;

- влияние организации на процессы устойчивого развития.

Она предполагает использование модели «встроенной безопасности», которая применяется для решения проблемы запаздывающего регулирования в быстро развивающихся областях деятельности, связанных с рисками для людей и окружающей среды. После анализа выявленных недостатков исходная модель была переработана для применения в целях устойчивости.

Ключевым звеном предложенного процесса «встроенной устойчивости» является использование совместной системы индикаторов устойчивости, риска и жизнестойкости. Это позволяет снизить влияние неопределенности и принимать максимально информированные решения в условиях запаздывающего регулирования.

Эту же систему индикаторов предлагается использовать в процессе формирования альтернатив компании в области устойчивости. Для этого предложен структурированный демократический процесс с участием заинтересованных лиц, основанный на четком определении ценностей компании и ее целей в области устойчивого развития. Такой подход может восполнить недостатки упрощенной модели индикаторов, заданной несколькими экспертами, и максимально эффективно использовать креативный потенциал заинтересованных лиц для формирования наиболее эффективной стратегии компании в области устойчивости.

\section{МИТЕРАТУРА}

1. Григорьева C.В. (2013). Оценка стратегической устойчивости в развитии предприятия // Вопросы экономики и права. Т. 3. С. 33-37.

2. Кузнецова М.О. (2020). Стратегическая устойчивость промышленных компаний: подходы к пониманию и анализ рисков // Стратегические решения и риск-менеджмент. Т. 11. № 2. C. 196-205. DOI: https://doi. org/10.17747/2618-947X-2020-2-196-205.

3. Самосудов М.B. (2006). Корпоративное управление: Теория корпоративного взаимодействия. Модульная серия «Экономист-международник». М.: ВАВТ Минэкономразвития России.

4. Саченко Л.А. (2020). Перспективы развития риск-менеджмента организаций с точки зрения общей теории систем // Проблемы анализа риска. Т. 17. № 4. С. 54-63. DOI: https://doi.org/10.32686/1812-5220-2020-17-4-54-63.

5. Afgan N.Y., Carvalho M.G., Hovanov N.V. (2000). Energy system assessment with sustainability indicators // Energy Policy. Vol. 28. P. 603-612.

6. Arena M., Azzone G. (2010). Process based approach to select key sustainability indicators for steel companies // Ironmaking and Steelmaking. Institute of Materials, Minerals and Mining. Vol. 37(6). P. 437-444.

7. Bruneau M., Chang S.E., Eguchi R.T., Lee G.C., O'Rourke T.D., Reihorn A.M., Shinozuka M., Tierney K., Wallace W.A., Winterfeldt D. von (2003). A framework to quantitatively assess and enhance the seismic resilience of communities // Earthquake Spectra. Vol. 19. P. 733-752.

8. Dos Santos F.T., Partidário M.R. (2011). SPARK: Strategic planning approach for resilience keeping // European Planning Studies. Taylor \& Francis Journals. Vol. 19(8). P. $1517-1536$.

9. Downes L. (2009). The laws of disruption: Harnessing the new forces that govern life and business in the digital age. Basic Books.

10. Faber M.H. (2018). On sustainability and resilience of engineered systems // Routledge handbook on sustainable and resilient infrastructure / P. Gardioni (ed.). Ch. 3. London: Routledge.

11. Ganin A.A., Massaro E., Gutfraind A., Steen N., Keisler J.M., Kott A., Mangoubi R., Linkov I. (2016). Operational resilience: Concepts, design and analysis // Scientific Reports. Vol. 6. No. 19540. DOI: 10.1038/srep19540.

12. Gillespie-Marthaler L., Nelson K., Baroud H., Abkowitz M. (2019). Selecting indicators for accessing community sustainable resilience // Risk Analysis. Vol. 11. Iss. 39. P. 2479-2498. 
13. Hardi P., Zdan T. (1997). Assessing sustainable development: Principles in practice. Winnipeg, Manitoba: Internation Institute for Sustainablie Development.

14. Lengnick-Hall C.A., Beck T.E., Lengnick-Hall M.L. (2011). Developing a capacity for organizational resilience through strategic human resource management // Human Resource Management Review. Vol. 21. P. 243-255.

15. Linkov I., Trump B.D., Hynes W. (2019). Resiliencebased strategies and policies to address systemic risks. Organisation for Economic Co-operation and Development. SG/NAEC (2019)5.

16. Linkov I., Trump B.D. (2019). The science and practice of resilience. Cham: Springer.

17. Marchant G.E., Allenby B.R., Herkert J.R. (2011). The growing gap between emerging technologies and legalethical oversight: The pacing problem. Dordrecht, Germany: Springer.

18. Mohamed E.S., Saleh A.M., Belal A.A. (2014). Sustainability indicators for agricultural land use based on GIS spatial modeling in North of Sinai-Egypt // The Egyptian Journal of Remote Sensing and Space Sciences. Vol. 17.

19. Nielsen L., Faber M.H. (2019). Impacts of sustainability and resilience research on risk governance, management and education // Sustainable and Resilient Infrastructure. DOI: 10.1080/23789689.2019.1611056.

20. Qien K., Massaiu S., Timmannsvik R.K. (2012). Guideline for implementing the REWI method. Trondheim, Norway: SINTEF. Report A22026.

21. Reid J., Rout M. (2020). Developing sustainability indicators - The need for radical transparency // Ecological Indicators. Vol. 110. No. 105941. DOI: https://doi.org/10.1016/j. ecolind.2019.105941.

22. Rose A., Krausmann E. (2013). An economic framework for the development of a resilience index for business recovery // International Journal of Disaster Risk Reduction. Vol. 5. P. 73-83.

23. Sachenko L. (2020). Organizational resilience. Cost optimization approaches. The 8th International Conference on Computing for Physics and Technology (CPT2020). Conference Proceedings. P. 138-143.

24. Sikula N.R., Mancillas J.W., Linkov I., McDonagh J.A. (2015). Risk management is not enough: A conceptual model for resilience and adaptation-based vulnerability assessments // Environment Systems and Decision. Vol. 35. P. 219-228.

25. Stewart R., Fantke P., Bjørn A., Owsianiak M., Molin C., Zwicky Hauschild M., Laurent A. (2018). Life cycle assessment in corporate sustainability reporting: Global, regional, sectoral, and company-level trends // Business Strategy and the Environment. Vol. 27. Iss. 8. P. 1751-1764.

26. Trump B.D., Keislerb J.M., Galaitsia S.E., PalmaOliveira J.M., Linkov I. (2020). Safety-by-design as a governance problem // Nano Today. Vol. 35. No. 100989. DOI: https://doi.org/10.1016/j.nantod.2020.100989.

27. и J., Handbook of Sustainable Management. London: Imperial College Press.

28. Yatsalo B., Sullivan T., Didenko V., Gritsyuk S., Mirzeabasov O., Pichugina I., Linkov I. (2005). Environmental risk management with the use of multi-criteria spatial decision support system DECERNS // International Journal of Risk Assessment and Management. Vol. 16. No. 4. P. 175-198.

\section{REFERENCES}

1. Grigoreva S.V. (2013). Otsenka strategicheskoy ustoychivosti $\mathrm{V}$ razvitii predpriyatiya [Assessment of strategic sustainability in enterprise development]. Voprosy ekonomiki i prava [Issues of Economics and Law], 33-37, 3.

2. Kuznetsova M.O. (2020). Strategicheskaya ustoychivost' promyshlennykh kompaniy: podkhody $\mathrm{k}$ ponimaniyu i analiz riskov [Strategic sustainability of industrial companies: Approaches to understanding and risk analysis]. Strategicheskie resheniya i risk-menedzhment [Strategic Decisions and Risk Management], 11(2), 196-205. DOI: https://doi.org/10.17747/2618-947X-2020-2-196-205.

3. Samosudov M.V.(2006). Korporativnoe upravlenie: Teoriya korporativnogo vzaimodeystviya [Corporate governance: Theory of corporate engagement]. Moscow, Russian Foreign Trade Academy of the Ministry for the Economic Development of the Russian Federation.

4. Sachenko L.A. (2020). Perspektivy razvitiya riskmenedzhmenta organizatsiy s tochki zreniya obshchey teorii sistem [Organizational risk-management perspectives from the general systems theory point of view]. Problemy analiza riska [Issues of Risk Analysis], 17(4), 54-63. DOI: https:// doi.org/10.32686/1812-5220-2020-17-4-54-63.

5. Afgan N.Y., Carvalho M.G., Hovanov N.V. (2000). Energy system assessment with sustainability indicators. Energy Policy, 28, 603-612.

6. Arena M., Azzone G. (2010). Process based approach to select key sustainability indicators for steel companies. Ironmaking and Steelmaking. Institute of Materials, Minerals and Mining, 37(6), 437-444.

7. Bruneau M., Chang S.E., Eguchi R.T., Lee G.C., O’Rourke T.D., Reihorn A.M., Shinozuka M., Tierney K., Wallace W.A., Winterfeldt D. von (2003). A framework to quantitatively assess and enhance the seismic resilience of communities. Earthquake Spectra, 19, 733-752.

8. Dos Santos F.T., Partidário M.R. (2011). SPARK: Strategic planning approach for resilience keeping. European Planning Studies. Taylor \& Francis Journals, 19(8), 15171536.

9. Downes L. (2009). The laws of disruption: Harnessing the new forces that govern life and business in the digital age. Basic Books.

10. Faber M.H. (2018). On sustainability and resilience of engineered systems. In: Gardioni P. (ed.). Routledge handbook on sustainable and resilient infrastructure. Ch. 3. London, Routledge.

11. Ganin A.A., Massaro E., Gutfraind A., Steen N., Keisler J.M., Kott A., Mangoubi R., Linkov I. (2016). Operational resilience: Concepts, design and analysis. Scientific Reports, 6, 19540. DOI: 10.1038/srep19540.

12. Gillespie-Marthaler L., Nelson K., Baroud H., Abkowitz M. (2019). Selecting indicators for accessing community sustainable resilience. Risk Analysis, 11(39), 2479-2498. 
13. Hardi P., Zdan T. (1997). Assessing sustainable development: Principles in practice. Winnipeg, Manitoba, Internation Institute for Sustainablie Development.

14. Lengnick-Hall C.A., Beck T.E., Lengnick-Hall M.L. (2011). Developing a capacity for organizational resilience through strategic human resource management. Human Resource Management Review, 21, 243-255.

15. Linkov I., Trump B.D., Hynes W. (2019). Resiliencebased strategies and policies to address systemic risks. Organisation for Economic Co-operation and Development, SG/NAEC (2019)5.

16. Linkov I., Trump B.D. (2019). The science and practice of resilience. Cham, Springer.

17. Marchant G.E., Allenby B.R., Herkert J.R. (2011). The growing gap between emerging technologies and legalethical oversight: The pacing problem. Dordrecht, Germany, Springer.

18. Mohamed E.S., Saleh A.M., BelalA.A. (2014). Sustainability indicators for agricultural land use based on GIS spatial modeling in North of Sinai-Egypt. The Egyptian Journal of Remote Sensing and Space Sciences, 17.

19. Nielsen L., Faber M.H. (2019). Impacts of sustainability and resilience research on risk governance, management and education. Sustainable and Resilient Infrastructure. DOI: 10.1080/23789689.2019.1611056.

20. Qien K., Massaiu S., Timmannsvik R.K. (2012). Guideline for implementing the REWI method. Trondheim, Norway: SINTEF. Report A22026.

21. Reid J., Rout M. (2020). Developing sustainability indicators - The need for radical transparency. Ecological Indicators, 110, 105941. DOI: https://doi.org/10.1016/j. ecolind.2019.105941.
22. Rose A., Krausmann E. (2013). An economic framework for the development of a resilience index for business recovery. International Journal of Disaster Risk Reduction, 5, 73-83.

23. Sachenko L. (2020). Organizational resilience. Cost optimization approaches. The 8th International Conference on Computing for Physics and Technology (CPT2020), Conference Proceedings, 138-143.

24. Sikula N.R., Mancillas J.W., Linkov I., McDonagh J.A. (2015). Risk management is not enough: A conceptual model for resilience and adaptation-based vulnerability assessments. Environment Systems and Decision, 35, 219-228

25. Stewart R., Fantke P., Bjørn A., Owsianiak M., Molin C., Zwicky Hauschild M., Laurent A. (2018). Life cycle assessment in corporate sustainability reporting: Global, regional, sectoral, and company-level trends. Business Strategy and the Environment, 27(8), 1751-1764.

26. Trump B.D., Keislerb J.M., Galaitsia S.E., PalmaOliveira J.M., Linkov I. (2020). Safety-by-design as a governance problem. Nano Today, 35, 100989. DOI: https://doi.org/10.1016/j. nantod.2020.100989.

27. Wu J., Wu T. (2012). Sustainability indicators and indices. In: Handbook of sustainable management. London, Imperial College Press.

28. Yatsalo B., Sullivan T., Didenko V., Gritsyuk S., Mirzeabasov O., Pichugina I., Linkov I. (2005). Environmental risk management with the use of multicriteria spatial decision support system DECERNS. International Journal of Risk Assessment and Management, 16(4), 175-198.

\section{ИНФОРМАЦИЯ ОБ АВТОРЕ}

\section{Лариса Анатольевна Саченко}

Кандидат экономических наук, генеральный директор ООО «Риск-профиль».

Область научных интересов: страхование, управление риском, экономика устойчивого развития.

E-mail: sachenko@risk-profile.ru

\section{ABOUT THE AUTHOR}

\section{Larisa A. Sachenko}

Candidate of economic sciences, CEO of "Risk-profile" LLC.

Research interests: insurance, risk management, sustainable development.

E-mail: sachenko@risk-profile.ru 